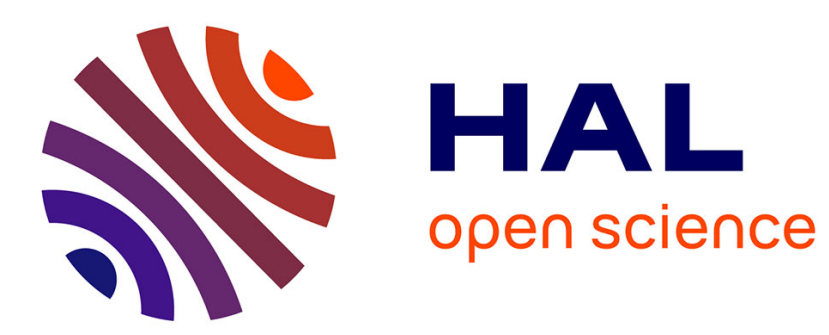

\title{
La politique de la ville intelligente en Chine : ancrage local d'un modèle urbain globalisé
}

Carine Henriot

\section{To cite this version:}

Carine Henriot. La politique de la ville intelligente en Chine : ancrage local d'un modèle urbain globalisé. Flux - Cahiers scientifiques internationaux Réseaux et territoires, 2018, 114 (4), pp.71. 10.3917/flux1.114.0071 . hal-02070719

\section{HAL Id: hal-02070719 https://hal.science/hal-02070719}

Submitted on 18 Jul 2019

HAL is a multi-disciplinary open access archive for the deposit and dissemination of scientific research documents, whether they are published or not. The documents may come from teaching and research institutions in France or abroad, or from public or private research centers.
L'archive ouverte pluridisciplinaire HAL, est destinée au dépôt et à la diffusion de documents scientifiques de niveau recherche, publiés ou non, émanant des établissements d'enseignement et de recherche français ou étrangers, des laboratoires publics ou privés. 


\section{LA POLITIQUE DE LA VILLE INTELLIGENTE EN CHINE : ANCRAGE LOCAL D’UN MODĖLE URBAIN GLOBALISÉ}

Carine Henriot

Université Paris-Est Marne la Vallée | « Flux »

$2018 / 4 N^{\circ} 114 \mid$ pages 71 à 85

ISSN 1154-2721

Article disponible en ligne à l'adresse :

https://www.cairn.info/revue-flux-2018-4-page-71.htm

Distribution électronique Cairn.info pour Université Paris-Est Marne la Vallée.

(C) Université Paris-Est Marne la Vallée. Tous droits réservés pour tous pays.

La reproduction ou représentation de cet article, notamment par photocopie, n'est autorisée que dans les limites des conditions générales d'utilisation du site ou, le cas échéant, des conditions générales de la licence souscrite par votre établissement. Toute autre reproduction ou représentation, en tout ou partie, sous quelque forme et de quelque manière que ce soit, est interdite sauf accord préalable et écrit de l'éditeur, en dehors des cas prévus par la législation en vigueur en France. Il est précisé que son stockage dans une base de données est également interdit. 


\title{
La politique de la ville intelligente en Chine : ancrage local d'un modèle urbain globalisé
}

\author{
Carine Henriot
}

L a ville intelligente ou smart city (1), en Chine comme ailleurs, s'inscrit au sein de l'urbanisme moderne (Picon, 2018), dans I'histoire des imaginaires urbains, comme l'un des multiples avatars de la ville du futur (Vanolo, 2014 ; Kitchin, 2014). L'expression smart résiste cependant à une définition universelle (Eveno, 2018). Incarnant la rencontre entre ville numérique et usages des technologies de l'information et de la communication par une communauté d'acteurs, la smart city revêt de "multiples facettes » (Albino, Berardi, Dangelico, 2015). La ville intelligente peut être appréhendée selon plusieurs approches : technologique, systémique et stratégique (Paroutis, Bennett, Heracleous, 2014). Si les approches technologique et systémique sont désormais relativement bien définies par les sciences pour l'ingénieur, nous positionnons cette contribution à l'articulation de l'approche stratégique, soit des jeux d'acteurs, et de l'approche spatiale de la smart city. Selon Angelidou (2014), I'approche spatiale de la ville intelligente correspond à un " modèle conceptuel de développement urbain basé sur l'utilisation du capital technologique, collectif et humain pour renforcer le développement et la prospérité des agglomérations urbaines » (p. S3). La ville intelligente, érigée en modèle, sert donc une finalité développementaliste et une intentionnalité. Loin d'être neutres, les discours porteurs $d^{\prime}$ intelligence, le smart urbanism et ses projets urbains, sont puissamment idéologiques et véhiculent une " utopie urbaine axée sur la technologie» (Hollands, 2015, p. 62). L'urbanisme intelligent auquel ses détracteurs reprochent d'être technocratique est pourtant loin d'être apolitique et non idéologique (Kitchin, 2015). Derrière l'affichage dépolitisé d'un projet smart, une mise en récit soignée assure aux multinationales pourvoyeuses de solutions technologiques de substantiels profits, ainsi qu'un marketing territorial ostentatoire aux municipalités qui accueillent ces projets innovants (Söderström, Paasche,
Klauser, 2014). Hollands (2015) souligne d'ailleurs «la nature auto-congratulatoire de la ville intelligente» (p. 63). Aussi, nous inscrivons cette analyse dans la perspective des travaux de Wiig et Wyly (2016), en nous intéressant à la smart city en tant que processus de transformation de la ville - chinoise -, en particulier de ses politiques d'aménagement urbain.

Ensuite, nous positionnons cette contribution sur les villes intelligentes chinoises à l'interface entre les approches spatiale et stratégique de la smart city, et la circulation, I'ancrage local et l'assemblage des politiques publiques (McCann, Ward, 2011 , 2012) urbaines et numériques dédiées à la production de démonstrateurs smart. Il existe en effet des éléments de convergence entre les caractéristiques internationales - voire globalisées - de la smart city, et son ancrage en Chine continentale, ses assemblages locaux, dont nous souhaitons rendre compte aux échelles nationales et métropolitaines. La smart city chinoise naît dans un contexte de crise économique entraînant une chute des exportations chinoises, un repli vers le marché intérieur, d'importants investissements en infrastructures et dans I'internet des objets (Wu et al, 2018), orchestré par le gouvernement central (Lu et al. 2015). Comme ailleurs, la smart city chinoise entend lutter contre les externalités négatives induites par l'urbanisation tardive et rapide que connaît le territoire (Cui, Wang, 2016), en améliorant le « système de systèmes urbains » (Wu et al., 2018). La ville intelligente chinoise trouve, comme ailleurs, ses origines dans la ville numérique (Lu et al., 2015), qui en Chine supplante la ville durable parmi les avatars de la ville du futur (Douay, Henriot, 2016). La smart city chinoise est également plurielle. Si la notion de ville intelligente est introduite en Chine continentale sous sa désignation anglophone, smart city, dans la seconde moitié des années 2000, d'autres termes sont parallèlement usités tout en désignant sensiblement le même type de projet, comme smarter city, smart pilot city, 
smart green city. Ces différentes terminologies fluctuent selon les technologies, les configurations d'acteurs mobilisés, et l'acception que chacun de ces acteurs a de la ville intelligente, pour se démarquer dans la compétition pour «l'intelligence ». Lorsque cette notion est appropriée et sinisée, la traduction officiellement retenue est alors: une "ville intelligente » (zhihui chengshi, 智慧城市). Elle se distingue, comme ailleurs, de la « ville numérique » (zhineng chengshi, 智能城市) par une gestion optimisée des systèmes informatiques et des services urbains qui les utilisent (réseaux d'électricité, de gaz, d'eau et assainissement, de transports), et par l'importance accordée à I'homme en tant qu'usager mais également en tant que citoyen au sein d'une "communauté intelligente» (zhihui shequ, 智慧社区), étroitement encadrée par les « comités de résidents 》 (shequ juminhui, 社区居委会). Ce dernier point souligne une spécificité locale : l'encadrement par le pouvoir local des smart cities chinoises et de leurs communautés d'usagers.

Dès lors, nous montrerons que les politiques de villes intelligentes chinoises témoignent d'une volonté de planifier le développement urbain à l'échelle nationale, pour orchestrer une modernisation rapide des équipements, des infrastructures et de la gestion des services urbains. Nous analyserons tout particulièrement la répartition des démonstrateurs intelligents sur le territoire, ainsi que les acteurs, les dispositifs de transfert et leur rôle respectif dans I'introduction, la normalisation, la diffusion de la notion smart, en rendant compte d'un ancrage local de ces politiques de ville intelligente.

Pour cela, nous nous appuierons sur les documents stratégiques et programmatiques de politiques numériques urbaines aux échelles nationale et municipales; les documents municipaux de planification urbaine; ainsi que les plaquettes promotionnelles et rapports produits par les entreprises, internationales, nationales et municipales, gestionnaires de services urbains, développeurs de solutions informatiques ou de technologies de l'information et de la communication se réclamant de la smart city. Ce corpus textuel sera complété par une analyse localisée. L'ancrage local de ces politiques de villes intelligentes sera analysé à travers trois initiatives municipales en Chine de l'Est. Un terrain réalisé à Shanghai au printemps 2018 a favorisé l'observation participante du recours généralisé au numérique dans la vie quotidienne; il alimente cette réflexion.

Nous montrerons comment les acteurs privés internationaux et chinois participent à l'introduction de ce nouveau modèle urbain en Chine continentale et à son ancrage local. Puis, nous nous intéresserons à l'encadrement par le pouvoir central de politiques publiques, fragmentées sectoriellement, promouvant un référentiel et le diffusant sur l'ensemble du territoire chinois. Enfin, nous rendrons compte du foisonnement des initiatives expérimentées en Chine de l'Est et de leur juxtaposition, à travers les exemples de Shanghai, Nankin et Hangzhou.

\section{Le RÔLE DES ACTEURS PRIVÉS dANS L'eXPÉRIMENTATION DE VILLES INTELLIGENTES en CHINe CONTINENTALE}

La ville intelligente chinoise est actuellement plus orientée vers l'usage de logiciels et applications (software) que vers le matériel informatique et la matérialité des réseaux (hardware) : elle passe, en effet, essentiellement par la téléphonie mobile et ses applications (Wu et al., 2018). Depuis 2012, le nombre d'utilisateurs de l'internet mobile a d'ailleurs dépassé le nombre d'utilisateurs d'internet sur PC (Luo, Wu, Yang, 2011). Cette transition d'une expérimentation du smart par le matériel informatique puis l'usage de logiciels s'effectue très rapidement, au bénéfice des entreprises chinoises et d'une diversification de ses acteurs. Les entreprises de l'électronique et de l'informatique, rapidement talonnées par celles de l'information, de la communication et des réseaux sociaux, sont à l'initiative de l'introduction de la ville intelligente en Chine continentale. C'est l'entreprise américaine d'informatique IBM qui la première investit le marché, avant d'être rapidement concurrencée par des entreprises chinoises de l'information, de la communication et des réseaux sociaux comme Huawei et Tencent. En effet, ces dernières ne se contentent plus d'imiter les technologies transférées; elles sont désormais capables d'innover. Pour la Chine, l'enjeu de la ville intelligente est ainsi de passer du Made in China au Innovated in China (Rochet, 2014, p. 14), le tout économiquement tiré par un marché intérieur de taille importante. On observe également une diversification de ces grandes entreprises porteuses d'innovation au sein des services à la ville. Parmi les secteurs émergents, l'entreprise chinoise de commerce en ligne Alibaba concurrence désormais les entreprises informatiques par sa capacité de stockage de serveurs, ce qui lui permet de se positionner dans la course au smart.

\section{IBM : fer de lance de " I'intelligence »}

En Chine continentale, l'entreprise américaine d'informatique IBM lance en 2008 un plan intitulé « De la percée de la ville intelligente en Chine » (Breakthrough of smart city in China). C'est alors par l'usage d'une terminologie anglo-saxonne, smart city, 
puis smarter city, dont IBM dépose la marque en 2009 pour gagner en lisibilité sur le marché naissant de la ville intelligente (Söderström, Paasche, Klauser, 2014), que cette entreprise internationale choisit d'investir le marché chinois. IBM organise, en 2009, un forum à Pékin structuré autour du slogan « éclairer une planète intelligente, construire une Chine intelligente » et destiné aux collectivités territoriales. IBM introduit alors I'informatique en nuage (2) qui doit permettre de dématérialiser le logiciel et de limiter les investissements en infrastructures. Les solutions IBM hébergées sur des plateformes en « nuages » sont mises en place dans cinq villes chinoises pilotes : Pékin, Shanghai, Shenzhen, Hangzhou et Wuxi. S'inscrivant au sein de grandes régions urbanisées, ces villes comptent toutes des parcs industriels reconnus pour la recherche et développement dans les NTIC. Pékin, Shanghai et Shenzhen correspondent en outre aux trois principales mégapoles chinoises, tandis que Hangzhou et Wuxi sont des villes moyennes de Chine de l'Est (cf. carte).

En 2011, IBM publie la plateforme Intelligent Operation Center (IOC) (3) laquelle propose sept solutions intelligentes pour la ville, dans les domaines du transport, de l'énergie, de l'eau et l'assainissement, de l'industrie, du logement, de la santé, et de la gouvernance. Parmi ces solutions, IBM se signale sur les secteurs de l'eau et l'assainissement, des transports, et surtout de la gouvernance pour la qualité de ses services de police et de sécurité.

En Chine de l'Est, le projet « une ville de Zhenjiang intelligente, pour un tourisme intelligent », lancé en 2012, constitue I'un des meilleurs démonstrateurs d'IBM en Chine continentale pour les solutions liées au transport intelligent (4). IBM a été choisie pour articuler le réseau de transports en commun par bus, ce qui concerne une flotte de 1000 véhicules, alimentant 80 lignes de bus et desservant 500 stations. Fin 2018, il est prévu que la flotte double pour atteindre 2000 véhicules répartis sur une centaine de lignes. Pour accompagner cette montée en puissance du réseau, la municipalité de Zhenjiang a contracté les solutions de transports intelligents proposées par la plateforme " transport public intelligent » d'IBM qui intègre des solutions de gestion du trafic, des aides aux voyageurs par de nouveaux médias, de la maintenance des équipements et de la gestion des actifs opérateurs.

Au-delà du cas de Zhenjiang, si c'est IBM qui a introduit le modèle de smart city par l'informatique en nuage et l'internet des objets (5), ses solutions connaissent un succès discret en Chine continentale, contrairement à la zone administrative spéciale de Hong Kong ou en République de Chine à Taiwan (6). En effet, les gouvernements locaux chinois souhaitent disposer d'un certain contrôle sur les données collectées, ce

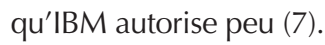

\section{Tencent et son application WeChat}

Parallèlement, les entreprises chinoises de NTIC se positionnent sur ce marché devenu concurrentiel, comme l'entreprise de téléphonie mobile Huawei ou encore l'entreprise Tencent. Créée en 1998 et implantée en Chine du Sud à Shenzhen, I'entreprise chinoise Tencent holding limited (8) est spécialisée dans les solutions de communication internet et mobile. En 1999, Tencent lance le système de messagerie instantanée propriétaire QQ, dont le fonctionnement se rapproche de MSN, tout en optimisant I'application originale, selon l'art chinois du shanzhai (9) ou de la « copie améliorée ». En juin 2016, la messagerie compte 899 millions d'utilisateurs. En 2011, Tencent lance I'application mobile gratuite de messagerie textuelle et vocale WeChat (weixin, 微信), destinée aux particuliers, qui compte, en mars 2018, plus d'un milliard d'utilisateurs et qui reste sans équivalent à ce jour en raison de l'interopérabilité des solutions proposées. Cette application fonctionne en effet comme une plateforme multiservices proposant, en plus des services classiques de messagerie textuelle et vocale, des services de jeux vidéo en ligne, de vente en ligne, le paiement sans contact, la gestion de son compte bancaire, ainsi que le transfert d'argent entre particuliers. Cette gestion intégrée de différents services, notamment bancaires, a bouleversé certains usages. Concrètement, la capacité de payer via son compte WeChat a complètement supplanté l'usage de l'espèce entre particuliers, de particuliers à professionnels, mais également entre professionnels. Dans les administrations, sur les campus universitaires, dans les entreprises, tous les services marchands sont désormais connectés à des comptes WeChat. Les utilisateurs ne payent plus en espèces ou par carte bancaire, mais via leur compte WeChat. Même les marchands de rue sont équipés de comptes WeChat. Désormais, en lieu et place des cartes bancaires ou d'espèces, tout un chacun scanne le QR-code du compte du particulier, de l'administration, de l'entreprise, du commerce de proximité ou de rue, auquel le quidam achète un produit ou un service; le vendeur renseigne le coût qui lui est dû ; l'acheteur présente son QR-code sur son smart phone, lequel est scanné par le vendeur; I'acheteur confirme l'achat, dont le montant est directement débité sur son compte bancaire. Plus besoin de recompter la monnaie. 
Devant le succès rencontré par la messagerie WeChat, devenue une véritable plateforme de services diversifiés et interopérables, I'entreprise Tencent lance, en décembre 2014 à Canton, une nouvelle fonction WeChat liée aux "services à la ville» (chengshi fuwu, 城市服务), destinée à intégrer globalement tous les services de la ville intelligente. L'utilisateur peut désormais, et ce, dans une quarantaine de municipalités chinoises à ce jour, se déplacer en ville en connaissant les horaires de transports en commun et l'état du trafic, prendre un rendez-vous médical, gérer son compte sécurité sociale ou son fond de prévoyance retraite, suivre la scolarisation de son enfant, effectuer des démarches administratives (demander son extrait d'acte de naissance, enregistrer son hukou auprès de la sécurité publique, effectuer une demande de passeport ou de visa interne), ou encore vérifier l'authenticité des factures qui lui sont présentées puis s'en acquitter... Concrètement, I'utilisateur de weixin chengshi fuwu n'a plus à multiplier les applications auprès de multiples enseignes marchandes et de services, les comptes clients, les identifiants et mots de passe associés : il rassemble, sur une seule et même application, adossée à un compte utilisateur unique, sa messagerie textuelle et vocale classique, son service météo et ses indicateurs de pollution, son service de trafic et de navigation, intégrant - efficacement - les transports en commun urbains, ferroviaires et aéroportuaires, son service d'achat en ligne, tous ses accès réseaux de jeux en ligne, un moyen de paiement aux professionnels et aux particuliers, et toutes ses démarches administratives auprès de services publics. Ce service complet de e-gouvernement est, semble-t-il (10), particulièrement performant sur l'intégration des solutions de sécurité publique, de santé et d'affaires sociales. Tencent s'oriente par ailleurs vers les professionnels pour concurrencer les autres entreprises de techniques de l'information (IT). En mars 2015, Tencent lance les services WeChat aux entreprises d'hôtellerie, de restauration, de billetterie, de stationnement, de commerce de détail, de santé, de campus universitaire... L'objectif de Tencent est ainsi d'étendre l'application du service "WeChat Pay » à l'ensemble du O2O (online to offline), soit de généraliser le recours à un canal numérique en ligne - I'application mobile "WeChat Pay »-pour acheter sur un canal physique - une boutique physique de biens ou de services.

L'entreprise Tencent a ainsi développé autour de son application WeChat des services aux particuliers dès 2011, aux municipalités en 2014 et aux professionnels en 2015. La vision intégratrice absolument inédite de cette plateforme de services diversifiés et interopérables, initialement dédiée à la messagerie textuelle et vocale entre particuliers, réalise la ville intelligente, par ses solutions tous azimuts et leur praticité, rendant l'application WeChat de plus en plus incontournable pour vivre ou travailler en Chine.

\section{Alibaba Cloud et son " cerveau de la ville »}

L'entreprise Alibaba (11) a été créée en 1999 à Hangzhou, en Chine de I'Est, et s'est spécialisée sur I'Internet à travers un marché public destiné à faciliter les échanges entre entreprises chinoises et internationales pour Alibaba, des plateformes de paiements et de ventes au détail - chinoise pour Taobao et internationale pour Aliexpress, un moteur de recherche dédié au magasinage, et plus récemment des services d'informatique en nuage. Cet acteur investit plus tardivement qu'IBM et Tencent le champ de la ville intelligente à travers son projet de «cerveau de la ville » développé à Hangzhou.

L'« Alibaba Cloud» (ali yun, 阿里云) (12), également appelé le «cerveau de la ville»(chengshi danao, 城市大脑) (13), est une solution d'informatique en nuage, en gestation depuis 2009 à Hangzhou (cf. carte). Le projet a été officiellement lancé le 13 octobre 2016, à l'occasion de la 18 exposition internationale du lac de l'est, lors de la conférence Hangzhou Yunxi 2016, conjointement organisée par la municipalité de Hangzhou et le groupe Alibaba. Cette conférence, qui constitue la manifestation la plus importante en Chine dans le domaine de l'informatique en nuage et des données massives, a été l'occasion de lancer officiellement la plateforme de partage de données appelée le "cerveau de la ville de Hangzhou ». L'objectif est d'aider les villes à penser leur développement par I'analyse de données et l'optimisation des services intégrés, tout en interagissant avec leurs citoyens. L'élaboration de cet outil d'aide à la décision réunit depuis plusieurs années les services municipaux de Hangzhou, à travers, entre autres, le Bureau de la police, le Bureau de l'administration des affaires urbaines, la Commission pour la construction urbaine et rurale, autour d'un consortium de treize entreprises, notamment : Alibaba pour la coordination du projet, via la fourniture d'une plateforme d'intelligence artificielle, Dahua Technology Co Ltd et Supcon pour les algorithmes de contrôle de la circulation du trafic et de la signalisation lumineuse, H3C et Foxconn pour leurs 500 serveurs dédiés aux calculs. L'architecture technique mise en œuvre est organisée autour de cinq systèmes : I'infrastructure informatique (1), I'acquisition des données (2) comprenant les sous-systèmes de production ou d'acquisition des données des 
entreprises partenaires, des données issues de l'internet des objets (IoT), des données générées par les téléphones mobiles des particuliers (devices mobiles), voire des données d'open data partagées par la municipalité, et les sous-systèmes d'acheminement des données vers la plateforme d'échanges de données (3) permettant l'agrégation et la transformation des données, ainsi que leur mise à disposition vers des systèmes décisionnels ou opérationnels, la fourniture de framework open-source (4) et la mise en œuvre d'applications (5) s'appuyant sur les données collectées et transformées, afin de faciliter l'analyse globale des besoins et d'optimiser la répartition automatique des ressources publiques, et ce, en temps réel.

L'entreprise, initialement de vente en ligne, est ainsi devenue un acteur de la ville intelligente, en lançant dès 2009, soit en même temps qu'IBM, son Alibaba Cloud, à l'origine du « cerveau de la ville» de Hangzhou.

Ainsi observe-t-on en Chine continentale une diversification des acteurs de la ville intelligente, à la faveur d'une montée en puissance des entreprises de l'information, de la communication et des réseaux sociaux comme Tencent ou de la principale entreprise de commerce en ligne, Alibaba, tandis que les entreprises d'informatique comme IBM, pourtant «à l'origine de l'agilité des services urbains » (Picon, 2018, p. 87), semblent connaître un développement plus limité. Loin des acteurs traditionnels de la gestion urbaine et des grands groupes internationaux, l'expérimentation du modèle de ville intelligente en Chine continentale s'est plutôt faite au bénéfice des acteurs chinois de la téléphonie mobile, ce qui constitue une originalité dans la manière de concevoir et de développer la ville intelligente.

\section{Des POlitiques PUbliques ENCADRANT ÉTROITEMENT L'ÉLABORATION ET LA DIFFUSION DES VILLES INTELLIGENTES CHINOISES}

Alors que les projets d'informatique en nuage et d'internet des objets portés par des entreprises privées sont en gestation, le gouvernement central chinois éprouve la nécessité d'encadrer la définition de la ville intelligente, d'expérimenter concrètement les solutions smart, puis d'en favoriser la diffusion sur le territoire chinois dans un souci de rééquilibrage territorial. En Chine continentale, le développement urbain et industriel fait l'objet d'une programmation contraignante, encadrée par une fabrique politique forte et centralisée, élaborée et validée lors d'expérimentations pilotes, structurée en objectifs déclinés à plusieurs échelles, et répliquée sur l'ensemble du territoire (Heilmann, Melton, 2013). Dès lors, la politique chinoise de villes intelligentes se caractérise par son caractère descendant (Lu et al., 2015); elle débute par une planification d'échelle nationale orchestrée par le gouvernement central (People's Republic of China, 2016), ce qui correspond plutôt à l'organisation institutionnelle et à la pratique chinoise de l'aménagement (Douay, Henriot, 2016). Cette vision programmatique de la planification urbaine souligne le fort encadrement étatique, dont les collectivités territoriales assurent le prolongement. Les principaux acteurs du gouvernement central chinois intervenant dans la programmation des politiques de villes intelligentes et leur diffusion au plan national sont la Commission pour les réformes et le développement, laquelle oriente et met en concurrence : le ministère du Logement et du Développement urbain et rural, le ministère pour la Science et la Technologie, le ministère de l'Industrie et des Technologies de l'Information, afin que chacun élabore son modèle de ville intelligente, couvrant ainsi l'ensemble des besoins identifiés.

\section{Une coopération entre UE et MIIT pour construire un référentiel commun}

En Chine continentale, la notion de ville intelligente est apprivoisée par l'État central et définie via un projet de coopération avec I'Union européenne (UE). Fin 2011, la Direction générale des Réseaux de Communication, du Contenu et des Technologies (CNECT) de la Commission européenne (14) et le ministère chinois de l'Industrie et des Technologies de l'Information (Ministry of Industry and Information Technology - MIIT) (15) convoquent la troisième réunion pour le dialogue sino-européen sur la technologie informatique. La coopération vise à établir un référentiel sino-européen d'expertise sur la ville intelligente pour favoriser la conception de "Green Smart City ». Cette appellation souligne en outre la filiation entre le paradigme de la ville durable et celui de la ville intelligente. Plus concrètement, des villes pilotes sont sélectionnées en Chine et en Europe, des indicateurs qualitatifs et quantitatifs sont élaborés et validés de part et d'autre, puis chaque initiative est l'objet d'une fiche synthétique permettant de mettre en évidence les principales caractéristiques " intelligentes » expérimentées, et de les comparer. Fin 2011, le MIIT promeut un programme quinquennal de développement de l'industrie de la sécurité de I'information, de l'Internet et du E-commerce dans le cadre de I'accord avec la Commission européenne sur les « Green Smart 
City ». En 2013, les coopérations d'experts techniques européens et chinois permettent la réalisation d'une étude comparative et la publication en 2014 d'un Livre blanc (EU-China, 2014). Quinze villes sont désignées comme site pilote (cf. carte) en partenariat avec une ville européenne (cf. tableau 1).

Les dialogues techniques nés de ces rapprochements doivent permettre de mieux comprendre les secteurs clés pour les deux parties prenantes et leurs enjeux, dans le but de renforcer les relations industrielles stratégiques entre l'Union européenne et la Chine. Les villes intelligentes chinoises élaborées dans le cadre de cette coopération bénéficient ainsi $d^{\prime}$ un échange d'expertise et d'un retour d'expériences smart européennes.

\section{Les ministères MIIT et MOST mis en concurrence et encadrés par la NDRC}

La Commission pour les réformes et le développement (National Development and Reform Commission - NDRC) (16), équivalent chinois du Commissariat au Plan français, et au-delà le gouvernement central nourrissent pour ambition d'élaborer un modèle chinois de ville intelligente. Ils mobilisent pour cela l'ensemble des acteurs à leur disposition en les positionnant en situation de concurrence. Ainsi, les initiatives se multiplient-elles. Dès 2012, le MIIT et la NDRC conçoivent et diffusent un document national promouvant le développement de villes intelligentes. Un avis est formulé quant à la réalisation de sites pilotes nationaux de villes intelligentes, avec la mise en

Tableau 1. Les villes européennes et chinoises, pilotes des " green smart cities »

\begin{tabular}{|c|c|}
\hline 15 villes pilotes EU & 15 villes pilotes chinoises \\
\hline Amsterdam - Pays-Bas & Arrondissement de Haidian - Pékin \\
\hline Barcelone - Espagne & Nouvel arrondissement de Binhai - Tianjin \\
\hline Bristol - Angleterre & Nouvel arrondissement de Pudong - Shanghai \\
\hline Copenhague - Danemark & Yangzhou - Jiangsu \\
\hline Florence/Prato - Italie & Nantong - Jiangsu \\
\hline Francfort - Allemagne & Ningbo - Zhejiang \\
\hline Issy-les-Moulineaux - France & Jiaxing - Zhejiang \\
\hline Lyon - France & Zhangzhou - Fujian \\
\hline Malmö - Suède & Yantai - Shandong \\
\hline Manchester - Angleterre & Arrondissement de Nansha - Canton \\
\hline Riga - Lettonie & Qianhai - Shenzhen \\
\hline Tallinn - Estonie & Nouvel arrondissement de Hengqin - Zhuhai \\
\hline Venise - Italie & Chengdu - Sichuan \\
\hline Vilnius - Lituanie & Korla - Xinjiang \\
\hline
\end{tabular}


avant de mesures provisoires et transitoires, pour démarrer les applications concrètes à l'échelle des districts et des municipalités. Le MIIT choisit de développer deux sites pilotes en Chine de l'Est: Yangzhou et Changzhou ( $c f$. carte). Parallèlement, le ministère pour la Science et la Technologie (Ministry of Science and Technology-MOST) (17) organise, en 2012, une alliance dédiée aux villes intelligentes, la " China Smart City Industry Alliance », et promeut deux sites pilotes : Shenzhen et Wuhan (cf. carte).

\section{Le temps de la diffusion orchestrée par le MOHURD}

En 2012, 2013 et 2014, la programmation change d'échelle avec la publication de trois appels d'offres successifs invitant les collectivités territoriales chinoises à présenter des projets leur permettant d'être labellisées comme site pilote de «ville intelligente 》 (zhihui chengshi, 智慧城市) par le ministère en charge des questions d'aménagement: le ministère du Logement et du Développement urbain et rural (Ministry of Housing and Urban-Rural Development-MOHURD) (18). À noter qu'en août 2014, la troisième phase de programmation de villes intelligentes correspond à une action commune au MOHURD et au MOST visant à regrouper les expertises des deux ministères. La mise en œuvre s'appuie sur l'implantation, à Pékin, d'un nouveau Centre national pour l'Innovation, mais fait aussi appel à la mobilisation de différents acteurs au niveau local, notamment pour financer ces projets par le partenariat public-privé.

Faisant suite à ces appels d'offres, 90 collectivités territoriales chinoises sont sélectionnées fin 2012, puis 103 en août 2013 et enfin 97 en avril 2015 ; ce qui fait un total de 290 démonstrateurs à construire en 3 à 5 ans. À l'issue de leur aménagement, le MOHURD évaluera l'ensemble des 290 villes pilotes et les classera en leur attribuant 1, 2 ou 3 étoiles. Ce programme vise à diffuser les initiatives smart dans les mégapoles, les grandes villes, mais également les villes moyennes chinoises, et ce, sur l'ensemble du territoire (cf. carte) ; à renforcer la capacité de planification des villes-centre au regard de leur périphérie ; à promouvoir les TIC comme outil de gestion urbaine, l'écologie et le bas-carbone comme nouveau mode de développement.

\section{De l'expérimentation à la standardisation}

Ainsi quatre acteurs du gouvernement central chinois interviennent dans l'expérimentation des politiques de villes intelligentes et leur diffusion, selon une programmation nationale.
Le MIIT a initié la coopération entre I'Union européenne et la République populaire de Chine. La NDRC intervient pour guider le MIIT dans ses premières actions, ajouter certains enjeux dans le giron des villes intelligentes, comme la question des transports et mobilités, puis articuler les différents ministères. Le MOST se positionne également sur le marché de la ville intelligente. Enfin, il incombe au MOHURD de programmer et de diffuser à grande échelle ces politiques de villes intelligentes, à travers l'implantation de sites pilotes dans toute la Chine.

Comme le souligne Leprêtre (2018, ce dossier), le recours croissant à l'expérimentation pour faire l'action publique tant du côté des collectivités territoriales que du côté de l'État est révélateur de la dimension démonstrative des expérimentations appropriées à la fois par les acteurs locaux et nationaux. Ces « bonnes pratiques » articulent également la coexistence d'une demande simultanée de standardisation et de différenciation chez les collectivités territoriales et les acteurs privés. Toujours selon Leprêtre (2018, ce dossier), le modèle se trouve alors plus dans la gouvernance des projets que dans les rôles assignés aux technologies. Il correspond ainsi à un instrument mobilisé par l'État pour encourager les territoires dans leur recherche de compétitivité économique, de marketing urbain, d'innovation et d'exemplarité, et les mettre en concurrence pour l'obtention de financements.

En 2014, renforçant ces différentes politiques ministérielles chinoises, un document national d'orientation, intitulé "National planning on new urbanisation (2014-2020) », réaffirme la poursuite des politiques et la construction de villes intelligentes, opportunité dont les collectivités territoriales se saisissent. Dès 2015, les différentes expertises et expérimentations ministérielles font l'objet d'une standardisation. En 2016, le " groupe de travail inter-ministériel pour la coordination de new smart cities » promeut la construction de 100 nouvelles expérimentations, portant alors le nombre de villes intelligentes chinoises à plus de 500 à l'horizon 2017-2018 (19).

La géographie des villes intelligentes chinoises révèle, à l'échelle nationale, leur localisation initiale en Chine littorale, soit dans les zones les plus urbanisées, industrialisées, développées, puis leur diffusion sur l'ensemble du territoire chinois, dans une logique de développement et d'équité territoriale. À l'échelle régionale, la géographie des villes intelligentes rend compte d'une structuration en cluster. Ces smart city clusters (Lu et al., 2015) correspondent à la multiplication de projets smart, comme c'est le cas en Chine de l'Est. 
Les villes intelligentes en Chine : démonstrateurs privés, nationaux et programmes de diffusion

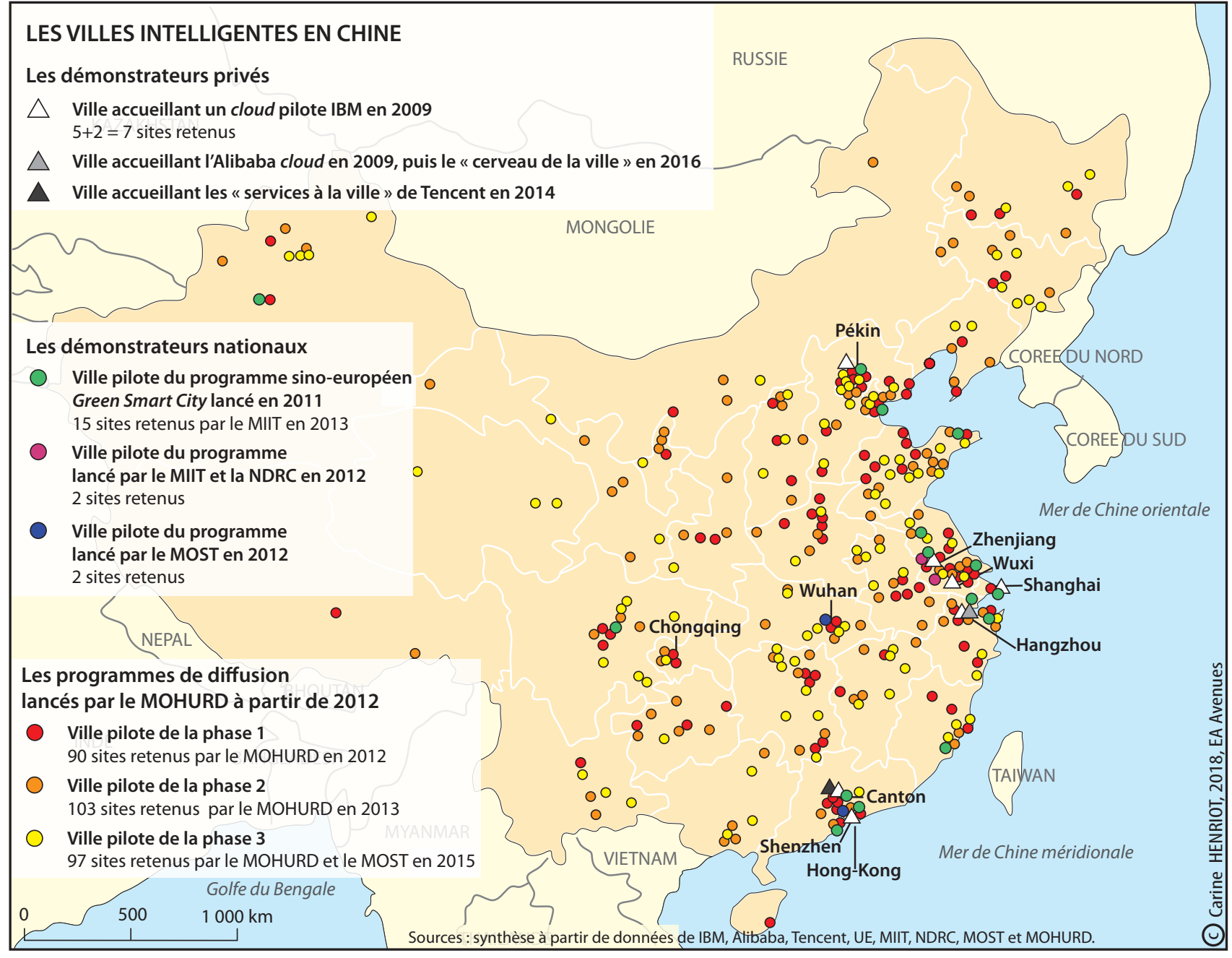

\section{Pour une Chine de l'Est résolument INTELLIGENTE : DES PROJETS SMART NOMBREUX, AMBITIEUX, MAIS JUXTAPOSÉS}

En Chine de l'Est, l'axe Nankin-Shanghai-Hangzhou-Ningbo (cf. carte) correspond à une zone densément peuplée (115 millions d'habitants sur moins de $100000 \mathrm{~km}^{2}$ ), industrialisée et urbanisée, concentrant les infrastructures de transports et formant un corridor de développement d'échelle régionale. L'ensemble de ce réseau urbain polycentrique et hiérarchisé structure la plaine deltaïque en mégalopole du bas Yangtsé, du nom du fleuve éponyme. Le territoire mégalopolitain ne s'organise pas uniquement autour de la métropole shanghaienne ; au-delà des périphéries de Shanghai, les centres secondaires comme Nankin ou Hangzhou ont leurs propres logiques de développement. Ce polycentrisme et la très forte concurrence territoriale qu'il induit expliquent la multiplication de projets pilotes smart dans la région (plus d'une quarantaine, cf. carte), mais également la faible coordination des initiatives entre elles, voire leur juxtaposition. Chaque centre secondaire se positionne dans la course à l'intelligence, pour bénéficier de retombées économiques supposées, et promeut pour cela son 
propre démonstrateur, lequel s'appuie sur un fort écosystème local dédié à l'innovation et aux nouvelles technologies. Les démonstrateurs les plus avancés relèvent ainsi de la convergence de trois facteurs se renforçant l'un, l'autre : des initiatives locales publiques et/ou privées, portées, voire programmées, par une municipalité forte (1), doublant le plus souvent les démonstrateurs de rang national (2), et s'appuyant sur un écosystème local, dynamique et fortement entreprenant (3).

\section{Nankin, ville pionnière de sa mise en intelligence}

À Nankin, la Municipalité semble prendre la mesure du tournant numérique dès 2006. Pour les politiques au pouvoir, l'intelligence correspond à la quatrième "vague d'innovation ", après l'industrialisation, l'électrification puis l'informatisation (Nankin, 2011). En décembre 2009, le comité municipal du parti et le gouvernement municipal de Nankin publient le projet stratégique "Nankin intelligente », où le concept d'intelligence est identifié comme un levier d'accélération de la modernisation urbaine et un moyen de prendre un tour d'avance dans la compétition territoriale que se livrent entre elles les villes de Chine de l'Est. Il faut toutefois attendre le douzième plan quinquennal et son document de référence, Planification générale du développement de la ville intelligente du douzième plan quinquennal (Nankin, 2011), pour que les objectifs de la construction soient planifiés avec en premier lieu, un développement orienté vers les réseaux à travers l'amélioration des infrastructures, puis dans un second temps, le développement orienté vers le logiciel pour l'industrie, l'e-gouvernement, l'e-commerce, l'e-service et en dernier lieu, la smart community. Dans cet exemple, Nankin illustre le montage pionnier d'un volontarisme municipal qui s'inscrit comme une déclinaison locale de la programmation nationale des smart cities, suivant une programmation technique d'abord orientée vers le hardware puis vers le software.

\section{Hangzhou, un démonstrateur privé ambitieux : l'exemple du système de gestion des transports et mobilités}

Le cas de Hangzhou est quant à lui emblématique d'une étroite collaboration menée entre l'entreprise Alibaba dont le siège est basé à Hangzhou, un tissu industriel et technologique entrepreneurial, composé en grande majorité d'entreprises privées, ce qui constitue une caractéristique de la province du Zhejiang, et une volonté municipale prête à transformer son territoire en démonstrateur de l'intelligence urbaine.
Comme nous l'avons vu dans la première partie de cette étude, si à l'origine IBM avait lancé en 2009 un « nuage » pilote, le projet s'est très vite vu concurrencé par I'« Alibaba Cloud », qui abrite le «cerveau de la ville» (chengshi danao, 城市大 脑). La plateforme portée par Alibaba, regroupant un consortium de 13 entreprises depuis 2009, situées au sein du hub technologique de la Hangzhou Hight Tech Development Area, est mise en fonction en octobre 2016. Tout comme Nankin, répondant ainsi à l'appel à projet national, la municipalité de Hangzhou (2011) publie un document de référence visant à stimuler la planification générale de la construction de la ville intelligente de la municipalité de Hangzhou. Pour l'heure, le " cerveau de la ville » se positionne sur l'optimisation des services urbains : le transport, l'énergie, l'eau et l'assainissement. En septembre 2016, un test préliminaire de transport intelligent dirigé par l'intelligence artificielle du « cerveau de la ville » a été effectué dans l'arrondissement de Xiaoshan à Hangzhou ; il a permis l'amélioration de la circulation routière de 3 à $5 \%$ en moyenne, avec une amélioration de $11 \%$ pour les secteurs les plus embouteillés, via la seule régulation « intelligente » des signalisations (20). Fin 2017, I'arrondissement de Xiaoshan à Hangzhou enregistre une amélioration de son trafic routier de l'ordre de $15 \%$, permettant de réduire les temps de trajets de 3 minutes. Les efforts entrepris courant 2018 portent désormais sur l'amélioration du trafic des services d'urgence comme les ambulances et les pompiers. En avril 2018, le temps de réponse des services d'urgence est diminué de $50 \%$, ce qui permet aux véhicules d'urgence d'arriver sur les lieux de l'accident en moyenne 7 minutes plus tôt (21). La plateforme informatique collecte et agrège pour cela aux informations SIG classiques de la ville : I'historique des positions GPS et en temps réel des bus, des taxis, des autres transports urbains, des véhicules particuliers, les données historiques des transporteurs, ainsi que tous les flux générés par l'internet des objets et la vidéo-surveillance (feux de signalisation, analyse du trafic des voies de communication par lecture de plaques minéralogiques...).

La ville semble ainsi s'être appropriée le projet, à l'origine privé, d'informatique en nuage, d'internet des objets et d'intelligence artificielle. Le couplage de I'«Alibaba Cloud» et du « cerveau de la ville » pour optimiser le trafic routier circule désormais en dehors de Hangzhou ; il a été introduit, à l'automne 2017, un an tout juste après son lancement à Hangzhou, dans les villes de Suzhou et Quzhou, Zhejiang, et sur l'île de Macao. Concrètement, ces services de gestion des flux de véhicules et 
leur régulation sont relativement proches des services développés à Singapour (22) dans le cadre du programme Smart Nation lancé en novembre 2014 (Kong, Woods, 2018). Toutefois, il conviendra de se montrer attentif au développement et à la gestion de cette smart city réalisée à l'initiative d'un grand groupe privé chinois. À ce jour, nous ne disposons d'aucune information quant aux modalités de partage des données massives récoltées par ce dispositif. Hangzhou et son « cerveau de la ville » constituent ainsi un démonstrateur grandeur nature des capacités d'innovation de l'entreprise de vente en ligne devenue un acteur de la gestion urbaine, mais au bénéfice de qui ? En effet, malgré l'affichage d'Alibaba qui articule son discours sur une ville intelligente au service de ses utilisateurs, force est de constater que l'habitant reste relativement discret dans le processus de «montée en intelligence ».

\section{À Shanghai, démonstrateurs privés, projets pilotes nationaux, municipaux et locaux se juxtaposent}

La métropole littorale de Shanghai est quant à elle emblématique d'un cas d'étude articulant une très forte volonté municipale, un démonstrateur de niveau national, porté par la municipalité et ses arrondissements, adossé à un tissu dense de conglomérats municipaux, d'entreprises innovantes chinoises ou internationales.

En effet, au dynamisme local encadré, dès 2009, par le «Three year action plan for Smart Pudong (2009-2011) », et réaffirmé au niveau municipal par le plan « Promoting Smart City Construction Plan (2011-2013) (Shanghai Municipal People's Government, 2013 ; Gil, Zheng, 2017), et par le plan de promotion du wifi « i-Shanghai »(爱上海, Ai Shanghai, littéralement « aime Shanghai ») lancé en 2012 (23), se surimposent

Tableau 2. Les programmes de ville intelligente à Pudong (Shanghai), une surimposition de programmes et d'acteurs

\begin{tabular}{|c|c|c|}
\hline Dates & Porteur & Programme de ville intelligente à Pudong \\
\hline 2009 & $\begin{array}{l}\text { Pudong et } \\
\text { Shanghai }\end{array}$ & Three year action plan for Smart Pudong (2009-2011) \\
\hline 2011 & Shanghai & Promoting Smart City Construction Plan (2011-2013) \\
\hline 2012 & Shanghai & i-Shanghai \\
\hline 2012 & MOHURD & Site pilote de la $1^{\text {er }}$ phase \\
\hline 2013 & UE-Chine & Site pilote «Green smart city» \\
\hline 2014 & Shanghai & Promoting Smart City Action Plan (2014-2016) \\
\hline 2015 & $\begin{array}{l}\text { Pudong et } \\
\text { Shanghai }\end{array}$ & $\begin{array}{l}\text { i-Pudong et sa campagne « 3-9-3-5 } \\
- \text { - } 3 \text { » programmes d'équipement en infrastructures (réseau de la fibre optique, wifi) } \\
- \text { « } 9 \text { » projets d'amélioration du système d'application du bien-être (services sociaux) } \\
\text { - «3» missions favorisant l'industrie } \\
\text { - « } 5 \text { » mesures principales de développement de l'environnement industriel }\end{array}$ \\
\hline 2016 & Shanghai & $\begin{array}{l}13^{\text {e }} \text { plan quinquennal de la municipalité de Shanghai } \\
\text { - e-gouvernement } \\
\text { - e-campus } \\
\text { - e-santé } \\
\text { - Smart-manufacturing (transition du « made in Shanghai » au «made by Shanghai ») }\end{array}$ \\
\hline
\end{tabular}


des initiatives relevant de l'État central (cf. tableau 2). Par exemple, en 2012, I'arrondissement de Pudong est sélectionné pour accueillir un projet pilote correspondant à la première phase de programmation de villes intelligentes planifiée par le MOHURD. De même, en 2013, l'arrondissement de Pudong fait partie des sites pilotes retenus par le projet de coopération sino-européen "Green Smart City ». En 2014, la municipalité de Shanghai, renouvelle le plan de promotion de la construction des smart cities. En 2015, le gouvernement d'arrondissement lance le programme «i-Pudong 2015 », lequel s'articule autour de projets bas-carbone, de transports publics intelligents, de services de santé intelligents et sur un projet de mise en place d'un e-gouvernement. Le principal slogan de i-Pudong est la « campagne 3-9-3-5 » (3935 zhanyi), articulée autour de « 3 » programmes d'équipement en infrastructures (réseau de la fibre optique, wifi), « 9 » projets d'amélioration du système d'application du bien-être (social), « 3 » missions favorisant l'industrie, et « 5 » mesures principales de développement de I'environnement industriel. Toujours à Pudong, quartier symbole du renouveau de Shanghai au début des années 1990, est lancé, dès 2009, le programme « Three year action plan for Smart Pudong (2009-2011) ». Le programme permet de solliciter les tissus d'entreprises implantés dans la Zone de nouvelles technologies de Zhangjiang, comme le géant chinois des technologies de la communication Huawei. Par un jeu de partenariats public-privé particulièrement rentables, pour 1 million de yuans investi par le gouvernement municipal et le gouvernement d'arrondissement de Pudong, les entreprises investissent 29 millions de yuans (24). Le recours au partenariat public privé, spécifiquement orienté vers la construction et la gestion de villes intelligentes, est par ailleurs désormais encouragé par le gouvernement central chinois dans le cadre du treizième plan quinquennal (2016-2020). Cette orientation est importante à prendre en compte parce que le précédent plan quinquennal (2011-2015), tout en encourageant le développement des smart cities selon une définition intégrative - les objectifs des villes numérique, durable, bas-carbone et créative étant assimilés dans la ville intelligente $-\mathrm{n}^{\prime}$ encourageait pas, à proprement parler, les investissements publics et privés vers le smart. Pudong bénéficie ainsi d'investissements et de programmes pilotes inscrits dans le champ de la ville intelligente, sans que les initiatives soient nécessairement articulées les unes aux autres.

La municipalité de Shanghai lance ainsi des programmes de promotion du numérique et des TIC, relayés par ses arrondissements et son tissu d'entreprises. Pour exemple, le projet
i-Shanghai lancé en 2012 prévoit l'implantation de 4000 bornes wifi en accès libre réparties sur l'ensemble du territoire municipal, afin de transformer la ville de Shanghai en large-scale wireless city. Toutefois, Wang et al. (2016) démontrent que « la ville sans fil » de Shanghai est réalisée, grâce à i-Shanghai, de manière très localisée dans le centre-ville, au centre d'affaires de Lujiazui, aux quartiers historiques et touristiques de Huangpu et du Bund, soit est restreinte aux principaux marqueurs urbains de la ville, tandis que les arrondissements de proche et de lointaine banlieue, les campus universitaires déconcentrés à Fangsong et Nanqiao, la zone de nouvelles technologies de Zhangjiang et le centre de recherche de Huamu à Pudong bénéficient, non pas du programme municipal i-Shanghai, mais de l'implantation de bornes de réseau local sans fil, par les compagnies téléphoniques privées. La " ville sans fil », programme phare de nombreux projets de villes intelligentes chinoises, est ainsi réalisée à Shanghai grâce aux investissements municipaux et privés, pour assurer une couverture voulue la plus ample possible. Cette géographie différenciée du wifi et l'origine des investissements consentis soulignent l'importance d'un marketing urbain prépondérant dans la politique publique de la Municipalité de Shanghai, et d'un pragmatisme certain de la part des opérateurs de téléphonie mobile.

Ainsi, la Chine de l'Est, en tant que mégalopole, se signale par la concentration et la juxtaposition des initiatives smart. Si les métropoles mondiales, comme Shanghai, jouent un rôle particulier de démonstrateur national, de site pilote local, puis de diffuseur de l'innovation vers d'autres espaces urbains, force est de constater que les grandes villes de Chine de l'Est, qui accueillent de nombreuses compétences R\&D en IT et en NTIC, ne restent pas en retrait, mais se positionnent elles aussi dans cette " course à l'intelligence ", comme Hangzhou qui exporte désormais son « cerveau de la ville » vers Hainan en Chine du Sud.

\section{Conclusion. RetOur SUR LA « MISE EN INTELLIGeNCE » DES VILLES CHINOISES}

Globalement, les politiques chinoises de villes intelligentes s'inscrivent dans une dynamique globalisée $d$ 'inflexion des modèles et référentiels urbains par un ancrage local. Cet assemblage est réalisé, d'un côté, par des multinationales étrangères comme IBM, mais surtout chinoises comme Tencent et ses « services à la ville » ou Alibaba et son « cerveau de la ville » - soit des acteurs 
du software, plus que du hardware - et, de l'autre côté, par des pouvoirs publics qui encadrent, à toutes les échelles, selon une planification urbaine développementaliste, programmatrice et descendante, ce nouveau leitmotiv des politiques urbaines. Les politiques de villes intelligentes chinoises sont d'abord alimentées par l'expérimentation, menée par la NDRC et plusieurs ministères (MOST, MIIT, MOHURD), standardisées via la reconnaissance d'initiatives labellisées entre 2012 et 2014, par le programme Smart Pilot Cities porté par le MOHURD, puis généralisées à partir de 2016, pour atteindre plus de 500 initiatives smart en 2018. Cette " mise en intelligence », orchestrée par le gouvernement central et répliquée à tous les échelons territoriaux, doit alors permettre un rattrapage territorial. La géographie des villes intelligentes chinoises se signale ainsi par une dissémination des initiatives sur l'ensemble du territoire et par une concentration des projets dans les mégalopoles de PékinTianjin, du delta du Yangsé et le delta de la rivière des Perles. Cette logique spatiale prévaut également à l'échelle infra-territoriale : chaque pôle d'une mégalopole et chacun de ses pôles secondaires dispose de sa smart city, tandis qu'une concentration des initiatives est observable à l'échelle intra-urbaine, où plusieurs projets peuvent être programmés, notamment dans les zones urbaines à haute valeur ajoutée, comme la zone de nouvelle technologie de Zhangjiang, sans pour autant être coordonnés entre eux. Cette impression de juxtaposition des projets smart est directement liée au fonctionnement des collectivités chinoises, subordonnées à un pouvoir fort, mais entretenant peu de relations entre elles, à un même échelon territorial. En effet, chaque niveau institutionnel de la sphère publique peut alors se retrouver en décalage, voire en concurrence. Localement, il se dessine alors une juxtaposition de configurations territoriales où acteurs privés et publics d'un même écosystème coopèrent, pour donner corps à cet engouement, certain en Chine, pour le numérique et sa « mise en intelligence ».

Ainsi la smart city chinoise se signale-t-elle par trois caractéristiques principales : le triptyque technologique (intelligence artificielle, données massives, internet des objets, ou Al, Big data, $(O T)$, une configuration d'acteurs publics et privés mobilisés pour l'innovation et la compétition territoriale, et un usage social prépondérant d'applications de téléphonie mobile. Cependant, ces démonstrateurs et projets restent faiblement connectés entre eux. Le principal défi des villes intelligentes chinoises sera donc, à défaut de rendre ses systèmes interopérables, de les articuler pour que le smart urbanism ne s'apparente pas à un urbanisme de projet, mais favorise la construction globale d'une ville chinoise voulue plus vertueuse. Au-delà, il convient de se montrer prudent quant à la mise en place de ces villes intelligentes. En Chine, où tout est sous contrôle de I'État, à commencer par les populations urbaines, quel usage les pouvoirs publics feront-il des données captées et exploitées en « nuage " ? Enfin, se pose la question de la circulation de la ville intelligente chinoise en dehors de Chine continentale. En effet, la smart city chinoise s'appuie sur des modèles prédictifs alimentés par de la biométrie, soit de la reconnaissance faciale, de l'analyse de vidéo, et ce, en temps réel... II n'est pas certain que dans la « course à l'intelligence » que se livrent entre elles les grandes puissances à forte capacité d'innovation, la Chine soit capable de transposer, en l'état, par exemple dans un contexte européen, au regard du Règlement Général sur la Protection des Données (RGPD), son modèle d'agglomération des données, aussi smart soit-il.

Carine Henriot est maître de conférences en aménagement de l'espace et en urbanisme, au sein du département de Génie urbain de Sorbonne universités, Université de technologie de Compiègne, chercheure à l'EA 7284 Avenues, et chercheure associée à I'UMR 8586 Prodig. Ses travaux portent sur la production de la ville chinoise et française, à travers la circulation de modèles urbains, les politiques d'aménagement et les pratiques de la planification territoriale, avec une focalisation sur le numérique et l'énergie. carine.henriot@utc.fr. 


\section{Notes}

(1) Nous choisissons de traduire la notion de smart city par ville intelligente et de les considérer comme synonymes, bien qu'en anglais la notion d'intelligent city renvoie au croisement de la société de connaissance avec la ville numérique (Yovanof, Hazapis, 2009), soit à une capacité de calcul favorisant l'innovation (Komninos, 2011 ; Woods, 2013), mais exclut la population et l'individu de sa définition (Woods, 2013) - au contraire de la smart city (Albino, Berardi, Dangelico, 2015). En mobilisant les expressions smart city et ville intelligente de manière interchangeable, nous nous inscrivons dans la ligne de Eveno (2018).

(2) L'informatique en nuage (cloud computing) consiste à proposer des services informatiques, mutualisés ou dédiés, pouvant aller de la fourniture de serveurs informatiques accessibles depuis internet et hébergés en dehors de l'entreprise accédant au serveur, à la fourniture de services informatiques (par exemple API). La facturation dépend en général de l'utilisation de la ressource et non de sa réservation.

(3) IBM, plaquette 1, " [IBM Intelligent City Platform - Intelligent Operations Management Center (IOC) Solutions] 智慧城市平台-智能运营管理中心 (IOC) 解决方案, IBM zhihui chengshi pingtai-zhineng yunying guanli zhongxin (IOC) jiejue fang'an », 2 pages. [En ligne] (consulté le 3 août 2018) Disponible à l'adresse : http://www-31.ibm.com/events/cn/2_ico.pdf

(4) IBM plaquette 2, « [IBM and Zhenjiang, Jiangsu together to build a “smart city»] IBM 携手江苏镇江共建 "智慧城市"， IBM xieshou jiangsu zhenjiang gong jian" zhihui chengshi" ", 2 pages. [En ligne] (consulté le 3 août 2018) Disponible à I'adresse: ftp://public.dhe.ibm.com/software/cn/casestudy/ A253199J71969Q36.pdf

(5) L'internet des objets (IoT ou Internet of Things) désigne les objets connectés.

(6) Anonyme, ingénieur informaticien chinois. Entretien réalisé par l'auteure le 17 mai 2018.

(7) ZHENG Feng, Professeur d'urbanisme et aménagement, Université de Nankin. Entretien réalisé par l'auteure le 27 mai 2015.

(8) Site officiel de l'entreprise Tencent, http://www.tencent. com/en-us/at/abouttencent.shtml (consulté le 3 août 2018).

(9) Selon Bosker (2013), la culture du « shanzhai » est un phénomène répandu et assumé en Chine. Le terme se réfère à la production de marchandises de marque contrefaites, reproduites au détail près, tout en offrant des caractéristiques supplémentaires, considérées comme manquant à l'original et destinées à répondre aux spécificités d'un marché donné.

(10) Nous aurions souhaité rendre compte du fonctionnement d'une smart community pilote à Shanghai. L'application portable "Minhang intelligent ", lancée en 2015, correspond en effet à un compte officiel WeChat et à ses "services à la ville» (chengshi fuwu, 城市服务), créé par l'entreprise Tencent, en collaboration avec le bureau d'arrondissement pour la Science et la technologie, déclinaison locale du Ministry of Science and Technology-MOST, et par I'entreprise municipale Shanghai Internet Software Co. Ltd. Cependant, n'étant pas résidente shanghaienne, enregistrée à la police locale, nous n'avons pas été en mesure de rejoindre le réseau social pour participer à la communauté intelligente des services à la ville de Minhang, et n'en avons observé le fonctionnement que par compte interposé.

(11) Site officiel de I'entreprise Alibaba, http://www.alibaba.com/ (consulté le 3 août 2018).

(12) Site officiel de l'Alibaba Cloud, https://www.alibabacloud.com (consulté le 3 août 2018).

(13) Présentation du projet de "Cerveau de la ville» à Hangzhou, dans un article de Laotie, publié le 16 octobre 2016. " [Hangzhou présente le cloud du «cerveau de la ville » ou comment améliorer la gestion urbaine] 城市大脑老 铁杭州发布“城市大脑”云计算如何助力城市管理; hangzhou fabu "chengshi danao" yun jisuan ruhe zhuli chengshi guanli ». [En ligne] (consulté le 3 août 2018) Disponible à l'adresse : http://laotie.baijia.baidu.com/article/657945

(14) Site officiel de la Direction générale des Réseaux de Communication, du Contenu et des Technologies (CNECT) de la Commission européenne, https://ec.europa.eu/info/departments/communications-networks-content-and-technology_en (consulté le 3 août 2018).

(15) Site officiel du ministère chinois de l'Industrie et des Technologies de I'Information (Ministry of Industry and Information Technology - MIIT), http://www.miit.gov.cn/newweb/ (consulté le 3 août 2018).

(16) Site officiel de la Commission pour les réformes et le développement (National Development and Reform Commission -NDRC), http://en.ndrc.gov.cn/ (consulté le 3 août 2018).

(17) Site officiel du ministère pour la Science et la Technologie (Ministry of Science and Technology - MOST), http:// www.most.gov.cn/eng/ (consulté le 3 août 2018).

(18) Site officiel du ministère chinois du Logement et du développement urbain et rural (Ministry of Housing and Urban-Rural Development-MOHURD), http://www.mohurd. gov.cn/ (consulté le 3 août 2018).

(19) Site officiel du groupe de travail inter-ministériel pour la smart city, http://www.smcstd.cn (consulté le 3 août 2018).

(20) Présentation du projet de "Cerveau de la ville » à Hangzhou, dans un article de Laotie, publié le 16/10/2016.

(21) Présentation du « cerveau de la ville » sur le site officiel Alibaba Cloud, https://www.alibabacloud.com/et/city ; Quelles sont les routines et tendances pour la construction des villes intelligentes?, Zixundingzhi, publié le 26 août 2017, [En ligne] (consulté le 3 août 2018) Disponible à l'adresse : https:// www.zixundingzhi.com/yunjisuan/f3e76a34f7b34b36.html

(22) Présentation des solutions de transport intelligent proposées à Singapour par SopraSteria. [En ligne] (consulté le 3 août 2018) Disponible à l'adresse : https://www.soprasteria. sg/en/markets/transport/sopra-steria-asia-wins-contract-withthe-land-transport-authority-(Ita)

(23) Shanghai Municipal People's Government, Présentation officielle du projet iShanghai. [En ligne] (consulté le 29 oct. 2018) Disponible à l'adresse: http://www.shanghai. gov.cn/shanghai/node27118/node27973/u22ai70898.html

(24) ZHENG Feng, Professeur d'urbanisme et aménagement, Université de Nankin. Entretien réalisé le 27 mai 2015. 


\section{Bibliographie}

Albino V., Berardi U., Dangelico R. M., 2015, Smart Cities: Definitions, Dimensions, Performance, and Initiatives, Journal of Urban Technology, Vol. 22, № 1, p. 3-21. DOI: 10.1080/10630732.2014.942092

ANGELIDOU M., 2014, Smart city policies: a spatial approach, Cities, n 41, S3-S11. DOI: 10.1016/j.cities.2014.06.007

Bosker B., 2013, Original Copies. Architectural Mimicry in Contemporary China, Hong Kong: Hong Kong University Press, University of Hawai'i Press.

Cui Q., Wang G., 2016, Driving Factors of Smart City Construction in China : An Exploratory Method by Grounded Theory and Empirical Research, Preprints. DOI: 10.20944/preprints201608.0210.v1

Douay N., Henriot C., 2016, La Chine à I'heure des villes intelligentes, L'Information géographique, n² 2016/3 (Vol. 80), p. 89-102. DOI : 10.3917/lig.803.0089

EU-China Smart and Green City Cooperation, 2014. Comparative Study of Smart Cities in Europe and China, Current Chinese Economic Report Series, Berlin: Springer.

EVEno E., 2018, La Ville intelligente : objet au cœur de nombreuses controverses, Quaderni, n 96, p. 29-41.

Gil O., Zheng T.-C., 2017, The Smart City Plan 2011-2013 in Shanghai, in: Jing Y., Osborne S. P. (eds), Public service innovations in China, Singapore: Palgrave

Hangzhou, 2011, Planification générale de la construction de la ville intelligence de la municipalité de Hangzhou, [杭 州市智慧城市建设总体规划, Hangzhou shi zhihui chengshi jianshe zongti guihua]. [En ligne] (consulté le 29 oct. 2018) Disponible à I'adresse: http://wenku.baidu.com/ view/6d8d37204b35eefdc8d33364.html

Heilmann S., Melton O., 2013, The Reinvention of Development Planning in China, 1993-2012, Modern China, 39(6), p. 580-628. DOI: $10.1177 / 0097700413497551$

Hollands R. G., 2015, Critical interventions into the corporate smart city, Cambridge Journal of Regions, Economy and Society, vol. 8, n 1, p. 61-77.

KITCHIN R., 2014, The real-time city? Big data and smart urbanism, Geo/ournal, 79, p. 1-14.

KıтCHIN R., 2015, Making sense of smart cities: addressing present shortcomings, Cambridge Journal of Regions, Ecnomy and Society, vol. 8, $\mathrm{n}^{\circ} 1$, p. 131-136.

Komninos N., 2011, Intelligent cities: variable geometries of spatial intelligence, Intelligent Buildings International, 3 (3), p. 172-188.

KonG L., Woods O., 2018, The ideological alignment of smart urbanism in Singapore: Critical reflections on a political paradigm, Urban Studies, vol 55 (4), p. 679-701.

LEPRÊTRE N., 2018 (ce dossier), Un « modèle national » de ville intelligente? Le rôle de l'État dans la mise en œuvre de réseaux électriques intelligents au Japon, Flux, 2018/4 (No 114), p. 9-21.

Lu D., Tian Y., Liu V.Y., Zhang Y., 2015, The performance of the smart cities in China - a comparative study by means of self-organizing maps and social networks analysis, Sustainability, 7, p. 7604-7621.
LuO J.Z., Wu W. J., YANG M., 2011, Mobile internet: terminal device, network and service, Chinese Journal of Computer [计算机, jisuanji], 8, p. 2029-2051.

McCann E., Ward K., 2012, Policy Assemblages, Mobilities and Mutations: Toward a Multidisciplinary Conversation, Political Studies Review, Vol. 10, No 3, p. 325-332.

McCann E., Ward K. (eds), 2011, Mobile Urbanism: City Policymaking in the Global Age, Minneapolis: University of Minnesota Press.

NankIN, 2011, Planification générale du développement de la ville intelligente du douzième plan quinquennal (20112015) de la municipalité de Nankin, [南京市“十二五”智慧城 市发展规划, Nanjing shi "shi'erwu" zhihui chengshi fazhan guihua]. [En ligne] (consulté le 3 août 2018) Disponible à I'adresse: http://www.jiangsu.gov.cn/jsgov/sx/shengxs/ nanjings/201212/t20121218309380.html

Paroutis S., Bennett M., Heracleous L., 2014, A strategic view on smart city technology: The case of IBM Smarter Cities during a recession, Technological Forecasting and Social Change, vol. 89, November, p. 262-272.

People's Republic of China, 2016, The 13th five-year plan for economic and social development of People's Republic of China (2016-2020). [En ligne] (consulté le 31 oct. 2018) Disponible à l'adresse: http://en.ndrc.gov.cn/newsrelease/201612/P020161207645765233498.pdf

Picon A., 2018, Villes et systèmes d'information : de la naissance de l'urbanisme moderne à l'émergence de la smart city, Flux, 2018/1 (No 111-112), p. 80-93.

Rochet C., 2014, Les villes intelligentes, enjeux et stratégies pour de nouveaux marchés, Étude réalisée avec le concours de CERGAM, CESAMES et du Service de coordination à l'intelligence économique (Ministère français des Finances et des comptes publics), Conference Paper, Conférence CMDM, Paris, novembre. DOI: 10.13140/2.1.4598.4962

Shanghal Municipal People's Government, 2013, Action Plan 2011-2013 of Shanghai Municipality for Building Smart City. [Online] (accessed 13 oct. 2017) Available at: http:// www.shanghai.gov.cn/shanghai/node27118/node27973/ u22ai70898.html

Söderström O., Paasche T., Klauser F., 2014, Smart Cities as corporate storytelling, City, Vol. 18, Issue 3, p. 307-320. DOI: 10.1080/13604813.2014.906716

Vanolo A., 2014, Smartmentality: The Smart City as Disciplinary Strategy, Urban Studies, Vol. 51, Issue 5, p. 883-998. DOI : 10.1177/0042098013494427

Wang M., Liao F. H., Lin J., Huang L., Gu C., Wei Y. D., 2016, The Making of a Sustainable Wireless City? Mapping Public Wi-Fi Access in Shanghai, Sustainability, 8(2), 111. DOI: 10.3390/su8020111

WIIG A., WYly E., 2016, Introduction: thinking through the politics of the smart city, Urban Geography, vol. 37 (4), p. 485-493.

Woods E., 2013, Smart cities. Infrastructure, information, and communication technologies for Energy, Transportation, Buildings, and Government: City and Supplier Profiles, Market Analysis, and Forecasts, New York: Pike Research. 
Wu Y., Zhang W., Shen J., Mo Z., Peng Y., 2018, Smart City with Chinese characteristics against the background of big data : idea, action and risk, Journal of Cleaner Production, 173, p. 60-66.

\section{Résumé - Carine Henriot - La politique de la ville intelligente en Chine : ancrage local $d^{\prime}$ un modèle urbain globalisé}

La ville intelligente ou smart city, en Chine comme ailleurs, se présente comme une utopie urbaine tournée vers la technologie. Cette contribution sur la politique de la ville intelligente en Chine se positionne à l'interface entre les approches spatiale et stratégique de la smart city, et la circulation de ce modèle urbain, à travers l'ancrage local des politiques publiques. Nous montrerons que les politiques nationales chinoises de la ville intelligente témoignent d'une volonté fragmentée de planifier le développement urbain à l'échelle nationale, pour orchestrer une modernisation rapide des équipements, des infrastructures, et de la gestion des services urbains. Nous montrerons également qu'introduits en Chine continentale par l'entreprise américaine d'informatique IBM, les démonstrateurs smart sont expérimentés, à la faveur d'une montée en puissance des entreprises chinoises de l'information, de la communication et des réseaux sociaux comme Tencent ou de la principale entreprise de commerce en ligne, Alibaba. Encouragées par l'obsession $d^{\prime}$ un rattrapage technologique endogène et des politiques nationales tous azimuts, les municipalités chinoises multiplient les initiatives smart, dans un contexte de recours généralisé au numérique, en particulier pour la vie quotidienne des populations chinoises. Chine

Mots-clés: ville intelligente, politique publique, NTIC,
Yovanof G. S., HazapiS G. N., 2009, an architectural framework and enabling wireless technologies for digital cities and intelligent urban environments, Wireless Personal Communications, vol. 49, n³, p. 445-463.

\section{Abstract - Carine Henriot - Smart city policy in China: local anchoring of a globalized urban model}

In China as elsewhere, the smart city is an urban utopia turned toward technology. This article on smart city policy in China is positioned at the interface between the spatial and strategic approaches of the smart city, and the circulation of this urban model, through the local anchoring of public policies. I demonstrate that the Chinese national policies of the smart city reflect a fragmented will to plan urban development at the national level, in order to orchestrate a rapid modernization of equipment, infrastructure and management of urban services. I also show that the smart demonstrators, introduced in mainland China by the American company, IBM, are experienced, with the rise of Chinese ICT companies and social networks like Tencent, or the leader in global online wholesale trade, Alibaba. Encouraged by the obsession with endogenous technological catch-up and all-out national policies, Chinese municipalities are multiplying smart initiatives, in a context of widespread use of digital technology by Chinese populations in their daily lives.

Keywords: smart city, public policy, ICT, China 